\title{
La transición del comportamiento alimentario: una explicación desde la teoría de la conducta*
}

Feeding Behavior Transition: An Explanation from Behavior Theory

\author{
Alma Gabriela Martínez Moreno** \\ Universidad de Guadalajara, México \\ Antonio López-Espinoza \\ Universidad de Guadalajara, México
}

\footnotetext{
**Centro de Investigaciones en Comportamiento Alimentario y Nutrición (CICAN). Centro Universitario del Sur. Universidad de Guadalajara. Av. Enrique Arreola Silva No. 883, colonia centro C.P. 49000. Ciudad Guzmán, Jalisco, México. Teléfono: +52 (341) 575 2222, Fax: 01 (341) 5752223. Correo electrónico: antonio_lopez_espinoza@hotmail.com
}

\section{RESUMEN}

La Teoría de la Conducta representa un medio para explicar la transición del comportamiento alimentario de los seres humanos, pues organiza los eventos psicológicos y clasifica cualitativamente las funciones estímulorespuesta. Por esta razón, el propósito del presente documento es abordar el proceso de transición del comportamiento alimentario, detallando las cinco funciones psicológicas propuestas por dicha teoría, con la finalidad de diferenciar los cambios conductuales de las prácticas alimentarias tradicionalmente explicadas en función de factores biológicos y culturales en la transición de la dieta. El análisis se organizó de tal modo que se distinguieron las dimensiones de los sistemas de alimentación respecto a las funciones psicológicas propuestas por Ribes y López en 1985.

Palabras clave

Teoría del Conducta, funciones psicológicas, comportamiento alimentario, seres humanos.

\section{ABSTRACT}

Behavior Theory represents a way to explain to human's feeding behavior transition. This theory organizes the psychologist's events and classifies stimulus-response functions through qualitative analysis. For this reason, the purpose of the present document is to approach the feeding behavior transition processes detailing the five psychological functions proposed by Behavior Theory. The target was to characterize the behavioral changes of feeding practices in diet transition that were explained through biological and cultural factors traditionally. The analysis was organized until way that feeding systems dimensions were distinguished to respect to the psychological functions proposed by Ribes and López in 1985.

Keywords

Behavior Theory, psychological functions, feeding behavior, human beings.

Para citar este artículo: Martínez Moreno, A. G., \& LópezEspinoza, A. (2016). La transición del comportamiento alimentario: una explicación desde la teoría de la conducta. Universitas Psychologica , 15 (4). http://dx.doi.org/10.1114 4/Javeriana.upsy15-4.tcae 
Una propuesta alternativa para analizar los eventos psicológicos es la Teoría de la Conducta de Ribes y López (1985), publicada hace 30 años, que se fundamenta en la Psicología Interconductual de Kantor (1978), que estudia la conducta desde un enfoque no mentalista, mediante procedimientos metodológicos que aseguren control y validez. Quizá, su planteamiento más original es la interconducta -una redefinición del término conducta- que refiere a la interacción de un organismo con las propiedades de su ambiente, subrayando una cualidad de interdependencia simultánea de varios factores que participan en él, conformando así una organización jerárquica compleja cuya finalidad es clasificar las funciones estímulorespuesta. En ese sentido, la Teoría de la Conducta propone una clasificación de distintos niveles del comportamiento como medio para entender estas interacciones del organismo con su entorno. Es posible entenderla como un instrumento específico para organizar conceptualmente los eventos psicológicos.

Ribes (1998) definió la Teoría de la Conducta como una clasificación cualitativa de las funciones estímulo-respuesta. Por su parte, Ribes y López (1985) denominaron como funciones psicológicas a cinco posibles niveles de interacción entre un organismo y el objeto de estímulo. Ribes (2007) señaló que el concepto de función se refiere a la organización de las relaciones estímulo-respuesta que se llevan a cabo dentro de un sistema de condicionalidades. Con base en lo anterior, en el presente trabajo se abordaron detalladamente las cinco funciones psicológicas descritas en la Teoría de la Conducta de Ribes y López (1985) para caracterizar el proceso de transición que ha sufrido el comportamiento alimentario del ser humano. La taxonomía (una organización para categorizar) propuesta sobre los niveles del comportamiento se relacionó con el trabajo de Mithen (1996) sobre los orígenes del ser humano, en su obra titulada The prehistory of the mind, donde -a pesar de que está enfocada al origen del arte, la ciencia o la religión- aborda de manera tácita una serie de acontecimientos que el autor considera decisivos para entender el comportamiento de los seres humanos, acontecimientos que también trastocaron y delimitaron la forma de alimentarse y, por ende, de sobrevivir. De manera específica se analizó el proceso de transición del comportamiento alimentario del hombre a través del sistema de condicionalidades de las interacciones estímulorespuesta.

En este sentido, el análisis se organizó de tal modo que se caracterizaron las dimensiones de los sistemas de alimentación respecto a las funciones psicológicas propuestas por Ribes y López (1985). Por último, se incluyeron cuestiones teóricas y metodológicas referentes a la variación de las prácticas alimentarias, explicadas en función de factores biológicos y culturales en la transición de la dieta del " homo ".

\section{Entendiendo la Teoría de la Conducta}

La base para comprender las premisas de la Teoría de la Conducta son los cinco niveles de organización del comportamiento denominados: 1) función contextual, 2) función suplementaria, 3) función selectora, 4) función sustitutiva referencial y 5) función sustitutiva no referencial. Percibir el aroma de una comida recién hecha representa la función contextual y corresponde al nivel más simple. En este caso, el organismo responde a los eventos sin alterarlos. Cuando un organismo responde y altera las relaciones entre eventos y estímulos, entonces la función ya es contextual: ponerle sal a la sopa porque está desabrida. En la función selectora el organismo también altera las relaciones entre eventos y estímulos al responder, pero a través de la mediación de otro evento, por ejemplo, voltear un trozo de carne de la parrilla al notar que ya está a término. En la función referencial sustitutiva una persona responde a través del lenguaje aún a pesar de que la situación o evento no estén presentes (es decir, ocurre lo que Ribes y López llaman un "desligamiento funcional"). Pedirle al mesero que describa su recomendación del día podría ejemplificar 
esta función: la comida no está presente, pero mediante el lenguaje el comensal es capaz de decidir si quiere o no ordenarla. Por último, la función sustitutiva no referencial establece que una persona responde convencionalmente desligado de objetos y eventos, así como de la situación presente. Planear la lista de alimentos que se necesitarán para elaborar las comidas de toda la semana requiere de este nivel de complejidad.

Así pues, podrá notarse que los cinco niveles de interacción van gradualmente de lo básico a lo complejo. De igual forma, las dos últimas funciones son exclusivas del ser humano, pues implican el lenguaje y el nivel de desligamiento funcional alcanza sus niveles máximos. Si aún no ha quedado claro el término de desligamiento funcional, imagine que son las dos de la tarde y usted no desayunó esta mañana. Alguien pasa a su lado y dice en voz alta que se comerá una deliciosa quesadilla. Usted empieza a salivar, aunque la quesadilla no esté presente. El estímulo de escuchar la palabra "quesadilla" ha fomentado su respuesta de salivación. Usted no se está comiendo la quesadilla, probablemente no se la coma. Tampoco puede comerse la palabra quesadilla, pero las circunstancias y su experiencia de comer quesadillas lo han conducido a experimentar el desligamiento funcional: "El desligamiento es esencial para definir la conducta psicológica o interconducta, pues lo que la distingue del comportamiento biológico es su plasticidad interactiva con el ambiente" (Ribes \& López, 1985, p. 58).

\section{Comportamiento alimentario y su transición}

Las diversas transiciones que ha sufrido el comportamiento alimentario de los humanos representan un proceso largo y gradual sin límites o direcciones establecidas. En este proceso, han existido acontecimientos decisivos que determinaron el presente alimentario de los seres humanos; uno de ellos fueron las prácticas alimentarias que facilitaron o dificultaron el comportamiento global de nuestros antepasados.
Por esta razón, la alimentación ha constituido una preocupación básica para todas las sociedades humanas. Específicamente, la previsión de la búsqueda de alimento conformada por una serie de eventos conductuales definidos (búsqueda, encuentro, elección, manipulación y consumo) representa una de las claves para estudiar las acciones encaminadas a la ingesta de alimento como meta a corto plazo para hacer más eficientes y maximizar los procesos biológicos (Leal, Rojas, \& Camarena, 2011). Adicionalmente, el estudio del comportamiento alimentario debe tomar en cuenta dos aspectos fundamentales: el espacio donde se evalúan las conductas involucradas y el tiempo durante el que se estudiarán tales conductas (Cabrera \& Maciel, 2009).

Por su parte, Ribes (1992, 1998) apuntó que el comportamiento se fundamenta en el marco de un ambiente ecológico y cultural determinado, en el que los organismos se ajustan a las características de los sistemas biológicos, la historia conductual y los cambios ambientales. Es por ello que, para realizar un análisis de la transición del comportamiento alimentario, deben examinarse las consecuencias funcionales de las dietas a través del tiempo. Esto es posible mediante investigaciones científicas que proporcionan un marco histórico para analizar: a) cómo operan $-\mathrm{u}$ operaban- los sistemas alimentarios (Milton, 1993); b) cómo se modifican bajo el impacto de los cambios ambientales (Andrews \& Martín, 1992) y c) cómo son afectados por el desarrollo de nuevas tecnologías para la localización, producción y elaboración de alimentos (Ulijaszek, 2002).

\section{Niveles de interacción en la Teoría de la Conducta}

Los cinco niveles de interacción entre un organismo y su ambiente, propuestos por Ribes y López (1985), pueden ayudar a organizar el análisis de la transición del comportamiento alimentario del ser humano. Los niveles forman parte de una taxonomía de funciones psicológicas basada en dos conceptos 
principales: la mediación de la relación de contingencia entre cada elemento de la función y el desligamiento funcional de la respuesta (Ribes, 2004). A continuación, se describirá cada una de ellas, caracterizando la transición del comportamiento alimentario.

\section{Función contextual}

Una función estímulo-respuesta es una forma de medición de las contingencias en la interacción de un organismo con su ambiente (Ribes \& López, 1985). Desde su aparición en la tierra, los seres vivos interactuaron con su entorno; quienes se adaptaron exitosamente a las condiciones fisicoquímicas, sobrevivieron; quienes no encontraron formas de interacción productivas, desaparecieron. Las respuestas de un organismo a partir de su relación con eventos externos e independientes, representan la primera función psicológica: la contextual. El medio de contacto es fisicoquímico. Ribes y López (1985) señalaron que la respuesta solo ocurre como consecuencia de otra condición en la forma de un evento de estímulo. Es decir, el ambiente regula la relación individuoambiente. Todos los animales tienen este tipo de relación como su entorno cuando acaban de nacer: la mayoría son alimentados y protegidos por sus madres, su actividad es pasiva. No obstante, es difícil imaginar cómo nuestro más antiguo antepasado interactuaba con su medio solo mediante una función contextual, pues para subsistir necesitaba solamente de la disponibilidad ecológica del alimento.

Milton (1993) argumentó que durante varios años se pensaba que los simios, monos y prosimios se hallaban inmersos en una bóveda tropical repleta de comida disponible, en la que solo les bastaba alargar la mano para alimentarse. Sin embargo, la observación directa de grupos de primates ha desmentido estas afirmaciones, pues estos animales constantemente adoptan nuevas estrategias conductuales para obtener alimento, alterando algunas de sus propiedades (Tutin, Fernández, Rogers, Williamson, \& McGrew, 1992; Whiten, Byrne, Barton, Waterman, \&
Henzi, 1992). Esta situación nos lleva a describir la siguiente función psicológica.

\section{Función suplementaria}

En esta función el organismo responde diferencialmente ante los eventos ambientales. Ribes y López (1985) afirmaron que hay una modificación de las relaciones temporalesespaciales, en las que el organismo modula su conducta a la variabilidad producida en el ambiente. Posiblemente, tanto el llamado "eslabón perdido" como los chimpancés tuvieron este tipo de interacción con su ambiente. Mithen (1996) mencionó algunas estrategias de los chimpancés para alimentarse, como arrancar las hojas de las ramas para utilizarlas como sonda para cazar hormigas y termitas. Estos animales también construyeron y utilizaron una amplia gama de instrumentos empleados en diversas tareas. Es decir, eran capaces de manipular objetos.

Mithen (1996) también señaló que el uso de instrumentos por parte del chimpancé está relacionado con la obtención de alimento. Agregó que estos animales tuvieron un tipo de inteligencia de la historia natural (entendida como un conjunto de procesos cognitivos destinados a procesar información sobre los recursos, las plantas, animales y materias primas). Ribes y López (1985) argumentaron que, en la función suplementaria, los organismos no se limitan a alterar el contacto con los objetos y eventos. De acuerdo a esto, los chimpancés eran capaces de iniciar la búsqueda de víveres, realizando desplazamientos dirigidos exclusivamente a determinadas zonas provistas de recursos alimentarios. Iniciaban la interacción organismo-ambiente, pero con las condiciones físicas presentes. Es decir, adoptaban una nueva estrategia alimentaria cuando no había disponibilidad ecológica de los alimentos, situación determinada a través de la clasificación de los frutos y plantas disponibles como "comestibles" o "no comestibles". Es este caso particular, los animales modificaban su 
interacción con el ambiente como medida para asegurar su dieta.

\section{Función selectora}

Una vez que los simios, monos y prosimios adoptaron nuevas estrategias alimentarias, fue necesario encontrar mejores lugares para alimentarse además de tener un conocimiento capaz de distinguir mejor lo comestible de lo no comestible. Las dietas tuvieron que modificarse a partir de la preparación de los alimentos. Los Australopithecus ramidus, anamensis y apheresis fueron capaces de producir numerosos útiles líticos.

Mithen (1996) advirtió que es probable que las lascas de piedra afiladas se utilizaran para cortar la piel y seccionar trozos de carne. Adicionalmente, se ha demostrado que estos antepasados consumían grandes cantidades de carne (Andrews \& Martin, 1992; Blumenschine, 1992). Por su parte, Mithen (1996) mencionó que numerosos yacimientos arqueológicos fechados entre hace 1.5 y 2 millones de años presentan grandes cantidades de fragmentos de huesos animales mezclados con instrumentos. Se supone que son restos de algunas comidas. Milton (1993) reportó que los australopithecus poseían molares robustos ideales para consumir dietas más "duras".

El nuevo comportamiento alimentario de estos antepasados sugiere que además de diferenciar entre grupos de alimentos, había algunos que eran preferidos sobre otros, como la carne. La obtención de este alimento tenía que llevarse a cabo de forma grupal para que fuera exitosa. Particularmente, la nueva estrategia constituyó una nueva organización de conducta: representa la diferencialidad reactiva del organismo a las relaciones ecológicas cambiantes. Esas son las características de la función selectora.

Ribes (2004) señaló que esta función se relaciona con áreas como el control instruccional, formas de imitación y de interacción social como la cooperación y la competencia. Su característica principal es el desligamiento de las propiedades fisicoquímicas de eventos particulares. Estas condiciones se aplican a las tareas en las que las condiciones de estímulo permiten funcionalmente la selección y son independientes de la forma de responder del organismo a los segmentos de estímulo que varían de momento a momento (Ribes \& López, 1985). La función es selectora a través de la variabilidad de las características del entorno, es decir, la conducta depende de la conducta de otros individuos.

Como se mencionó anteriormente, es probable que la carne fuera una parte regular de la dieta de los antepasados y más exclusiva de los primeros homo . Mithen (1996) señaló que el paso a una dieta más rica en carne habría requerido una capacidad más sofisticada para predecir el emplazamiento de los recursos que la de los australopithecus, que eran predominantemente depredadores. En cambio, los primeros homo parecían comprender los beneficios de la vida en grupos y de la distribución de terrenos en búsqueda de mejores alimentos. Según Mithen (1996), el Homo habilis prefería vivir en grupos grandes en los que la búsqueda de lotes de comida se hacía individualmente o por parejas para luego compartir el alimento con el resto del grupo. Es decir, había cooperación entre los miembros del grupo que se reflejaba en una dieta más rica y con consecuencias postingestivas nutricionales positivas. En este sentido, Rozin (1995) señaló que la búsqueda, preparación e ingestión de los alimentos se convirtió en una ocasión social.

\section{Función sustitutiva referencial}

Las pautas de cooperación entre los primeros homos se complejizaron con la aparición del lenguaje. En este caso, la interacción se da entre individuos en forma de una reactividad conjunta en relación con el lenguaje. Este último es el elemento principal de la función sustitutiva referencial (Ribes \& López, 1985).

Mithen (1996) realizó algunos apuntes sobre el lenguaje de los humanos primitivos. Señaló que tenían capacidad lingüística porque su aparato vocal difería de la de otros animales. 
Aiello y Dunbar (1993, citados por Mithen, 1996) mencionaron que la capacidad lingüística apareció con el género Homo, hace unos 25.000 años a partir de la interacción social. El Homo habilis estaba orgánicamente equipado para desarrollar el lenguaje, al igual que el Homo erectus. Mithen (1996) sugirió que tenían una capacidad linguística limitada y empleada básicamente para la integración de las áreas técnica, natural y social.

Posiblemente, estas habilidades les permitieron enfrentar las condiciones climáticas desfavorables, y sobre todo, acceder a una nueva forma de aprovechamiento de recursos. Los primeros homos organizaron exitosas formas de distribución de los alimentos mediante reglas simples relacionadas con las circunstancias ambientales. Este es el caso de la alimentación de mujeres embarazadas o en lactancia y de la correspondencia de los alimentos recibidos de acuerdo a la actividad que un individuo ejercía dentro del grupo (Messer, 1995).

Otro ejemplo para caracterizar la función sustitutiva referencial es el caso de los neandertales . Fueron cazadores y saquearon animales muertos (por depredadores o por muerte natural), sobrevivían a base de la caza y carroñeo con un limitado avance tecnológico. Vivían en grandes grupos con una buena comprensión de su medio y de los animales que habitaban en él. Mithen (1996) señaló que poseían una inteligencia de historia natural avanzada. Una de sus herramientas básicas para alimentarse era la planificación para organizar la caza mediante la comprensión del comportamiento animal, así como saber acorralar a la presa en situaciones desfavorables, lo cual no sería posible sin algún grado de cooperación social. Por su parte, el reparto de alimentos pudo ser pauta dominante en la sociedad neandertal primitiva, dado que los recursos alimentarios habrían llegado por lo general en forma de grandes lotes (animales enteros muertos).

Mithen (1996) sugirió que la dieta de las madres lactantes neandertales, tuvo que ser de alta calidad para atender las demandas alimentarias de sus crías, por lo que eran las primeras en recibir carne. Por su parte, Milton (1993) señaló que los humanos primitivos buscaron incorporar a su dieta diversos tipos de carnes para satisfacer sus requerimientos energéticos. La interacción de los neandertales fue entonces sustitutiva referencial, pues respondían y producían estímulos respecto a eventos no presentes en la situación.

Al responder a eventos ya ocurridos en otras situaciones, les permitió agilizar sus estrategias de distribución y consumo de comida, dando lugar a hábitos alimentarios dependientes de situaciones de estímulo particulares. La conducta alimentaria fue mediada por la conducta de otro individuo. $\mathrm{Y}$ ese individuo mediador responde a las mismas relaciones que el individuo mediado (Ribes, 1992).

\section{Función sustitutiva no referencial}

A pesar de los avances de los neandertales y de los primeros homos, sobre todo desde la aparición del lenguaje, hay una barrera sólida entre estos y el homo sapiens. Mithen (1996) argumentó que esta barrera se erige a partir de la ausencia de útiles para la ornamentación corporal y de ritos funerarios por parte de los miembros heridos del grupo, al parecer, tenían una limitada interacción social. Por su parte, los homo sapiens mostraron grandes progresos en esta y muchas otras áreas. Las evidencias de Mithen demostraron que con la llegada del Homo sapiens hubo una explosión cultural traducida en la fabricación de numerosos objetos destinados a la ornamentación personal como: anillos, collares y dientes perforados de animales. También aparecieron otras expresiones culturales como la pintura, la escultura y la música. Este crecimiento se llevó a cabo a pesar de que las condiciones ambientales no eran satisfactorias.

Este comportamiento es el que Ribes y López (1985) incluyen en la función sustitutiva no referencial, llamada así porque la relación se basa en eventos puramente convencionales. Se distingue por la total autonomía de la reactividad respecto a los eventos biológicos 
y fisicoquímicos. Sus características son: 1) la trascendencia de la situacionalidad de objetos y eventos, como el arte y la ciencia y 2) la sustitución de la realidad fisicoquímica y biológica por su sistema de contingencias basado en creaciones completamente humanas como los mitos, supersticiones y prejuicios.

Respecto al comportamiento alimentario, la aparición de la agricultura tuvo un fuerte impacto sobre la interacción entre el hombre y su ambiente alimentario. Aunque la forma en la que se llevó a cabo la introducción de la agricultura y el pastoreo no es muy clara. Mithen (1996) argumentó que los posibles factores que llevaron a su aparición hicieron posible la transformación de un cazador-recolector a un pastor-agricultor. Dicha transformación trajo grandes consecuencias alimentarias.

Ross (1995) señaló que la diferencia entre la dieta de los cazadores-recolectores y la de los pastores-agricultores se basa en las fuentes calóricas. La recolección de diferentes semillas y plantas, así como las actividades de caza y pesca incluyen un fuerte gasto de energía de una dieta que no siempre está segura. En cambio, la actividad de los pastores-agricultores incluía el sedentarismo y el aseguramiento de la fuente de alimentación. Agregó que la transición a la agricultura trajo algunas desventajas, sobre todo para la distribución de los recursos producidos, lo que se traduce en dietas empobrecidas. Las estrategias de distribución tuvieron que modificarse dando lugar a la aparición de mitos referentes a las prácticas alimentarias. Impedir en distintos grados el acceso a la comida entre las categorías de individuos (mujeres, hombres, viejos, niños, etc.), a partir de prohibiciones creadas por instituciones sociales resultó una estrategia exitosa para regular la disponibilidad ecológica del alimento.

La distribución de comida basada en mitos y tabúes también se ajustó a las relaciones de estatus y poder. Mithen (1996) describió las similitudes entre el comportamiento alimentario de un grupo de profesores de una universidad y el de una tribu africana: los dos grupos tiene alimentos representativos de poder y la distribución de la comida refleja claramente la posición de cada miembro dentro de su grupo social. Es decir, los alimentos se seleccionan en función de ciertas predilecciones religiosas, tribales o de otro tipo (Ross, 1995).

Por otro lado, Messer (1995) señaló que, en las sociedades occidentales, las concepciones sobre la "buena" o "mala" alimentación también pueden basarse en ideologías. La comida favorita de un grupo de individuos puede ser rechazada por otro. Los gustos y aversiones no son gratuitamente neutrales, sino que siempre parecen involucrar la afiliación de clase social, de religión y otras. Rozin (1995) mencionó que los factores socioculturales son más importantes para la selección de los alimentos que la posibilidad de ingerir los nutrientes adecuados.

Por último, el desarrollo de técnicas para la producción y elaboración de alimentos ha beneficiado su disponibilidad, aunque no sean nutricionalmente adecuados. Esta situación tiene por efecto aumentar el repertorio culinario y disminuir la repetividad alimentaria (Fischler, 1995). Sin embargo, es innegable que la gastronomía ha dado lugar a excelentes productos considerados como "arte culinario". Actualmente, el término cuisine se aplica para referir las reglas culinarias acerca del orden específico de preparación de ciertos platillos que incluyen el número de ingredientes, los sabores característicos y los modos particulares de preparación (Rozin, 1995). 


\section{TABLA 1}

Descripción de las funciones psicológicas en relación a la transición del comportamiento alimentario

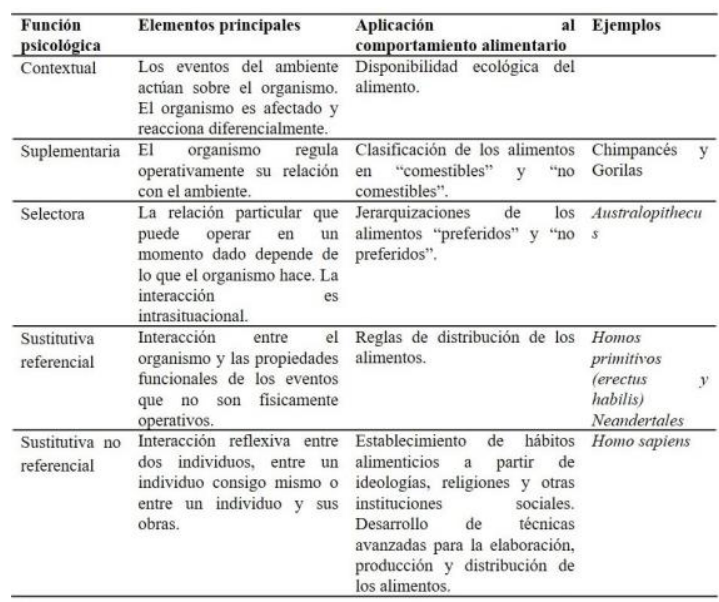

Nota:Se caracterizan las cinco funciones psicológicas propuestas por Ribez y López (1985) en la transición del comportamiento elimentario. Fuente: elaboración propia

\section{Conclusiones}

Durante más del $99 \%$ del tiempo transcurrido desde su aparición, el Homo sapiens vivió de la caza y la recolección. No es obstinado considerar que un buen número de características filogenéticas fueron seleccionadas en el curso de este periodo de la evolución humana. Este es, sin duda, el caso de la función de ciertos tipos de ecosistemas y de ciertos modos de interacción con el ambiente. Es decir, el caso de la función alimentaria.

Desde el punto de vista biólogico, el homo sapiens del neolítico comparativamente con el de la revolución industrial ha cambiado muy poco, pero en el plano cultural y sobre todo en sus interacciones con su ambiente se observan transformaciones profundas. ¿Qué sigue? Según Fischler (1995), la propensión a la obesidad podría ser fruto ni más ni menos que de la selección natural. Los obesos serían considerados como ahorradores de energía y capaces de acumular calorías en forma de grasa como medio para defenderse ante un cambio climático desfavorable que tenga como consecuencia la escasez de los alimentos. En cambio, los delgados serían "despilfarradores" de energía que queman sus calorías en lugar de almacenarlas. Sin duda, esta interpretación es ampliamente discutible, pero nos deja claro que el régimen alimentario actual es una decisión individual.

Por otra parte, Messer (1995) señaló que la estructura, el contenido y el cambio de la dieta son generalmente analizados con fines culturales o nutricionales. A un nivel más "popular", las costumbres alimentarias se han estudiado en términos de la formación y la persistencia de la cocina cultural (entendida como un conjunto de prácticas relacionadas con la alimentación, culturalmente elaboradas y trasmitidas de generación en generación). Sin embargo, los acontecimientos gastronómicos también pueden examinarse dentro de un marco interpretativo interconductual, donde la conducta alimentaria sea evaluada a modo de un proceso regulador ecológico y se incluya el estudio de determinadas variables. Por ejemplo: 1) la selección de un conjunto básico de alimentos (principales o secundarios); 2) el uso frecuente de un conjunto determinado de sabores; 3) la elaboración de estos alimentos; 4) la adopción de una variedad de reglas referentes a la aceptabilidad y combinación de alimentos, las comidas festivas, el contexto social de las comidas y los usos simbólicos de los alimentos y 5) las tendencias dietéticas en relación con la transición cultural.

El análisis psicológico sobre la transición del comportamiento alimentario de los seres humanos debe considerar la dieta junto con la conducta alimentaria como la parte básica para el estudio de las consecuencias sanitarias y medioambientales a las adaptaciones ecológicas. Cabe señalar que el análisis actual del comportamiento alimentario debe realizarse minuciosamente desde un contexto social. Ribes (2009) puntualizó que todo acto de comportamiento tiene sentido solo en términos del contexto social (histórico-cultural) en que ocurre. ¿Podrá ser reversible la transición del comportamiento alimentario? En su trabajo, Mithen (1996) señaló que en estas acciones 
no es una opción volver al diseño inicial para empezar otra vez desde el principio. De cierto modo, los sistemas alimentarios operan similarmente en varias especies, pero su comportamiento alimentario se ha modificado, haciéndose cada vez más complejo. Se convierte así en una interdisciplina que debe ser estudiada por especialistas en forma vertical, siendo las ciencias de la conducta el lugar protagónico en esta organización jerárquica. El comportamiento alimentario entendido como una interdisciplina debe dejarse de analizar como una actividad básica de naturaleza únicamente biológica. Solo entonces, será posible inferir de forma más contundente el efecto de los cambios sufridos en la alimentación de nuestros antepasados sobre el comportamiento alimentario reciente. No somos lo que comemos, somos los que comimos .

\section{Referencias}

Andrews, P., \& Martin, L. (1992). Hominoid dietary evolution. En A. Whiten \& E. M. Widdowson (Eds.), Foraging strategies and natural diet of monkeys, apes and humans (pp. 39-49). Nueva York: Clarendon Press Oxford.

Blumenschine, R. J. (1992). Hominid carnivory and foraging strategies, and the socioeconomic function of early archaeological sites. Philosophical Transactions of the Royal Society, 334, 211-221.

Cabrera, F., \& Maciel, R. P. (2009). Búsqueda e ingesta de alimento en el laboratorio operante: economías abiertas y cerradas. En A. López-Espinoza \& K. Franco (Eds.), Comportamiento alimentario: una perspectiva multidisciplinar (pp. 65-77). México: Editorial Universitaria.

Fischler, C. (1995). Gastro-nomía y gastroanomía: sabiduría del cuerpo y crisis biocultural de la alimentación contemporánea. En J. Contreras (Ed.), Alimentación y cultura (pp. 27-81). Barcelona: Universitat de Barcelona.

García, O., Rojas, M., \& Camarena, H. O. (2011). El estudio de la búsqueda de alimento desde una aproximación multidisciplinar: ecología conductual y análisis experimental de la conducta. En A. López-Espinoza \& A. G. Martínez (Eds.), Del hambre a la saciedad. Contribuciones filosóficas, psicológicas, socioantropológicas y biológicas (pp. 132154). México: Editorial Universitaria.

Kantor, J. R. (1978). Psicología interconductual . México: Trillas.

Leal, O., Rojas, M., \& Camarena, H. O. (2011). El estudio de la búsqueda de alimento desde una aproximación multidisciplinar: ecología conductual y análisis experimental de la conducta. En A. López-Espinoza \& A. G. Martínez (eds.), Del hambre a la saciedad. Contribuciones filosóficas, psicológicas, socioantropológicas y biológicas (pp. 132-154). Guadalajara, México: Editorial Universitaria. Universidad de Guadalajara.

Messer, E. (1995). Perspectivas antropológicas sobre la dieta. En J. Contreras (Ed.), Alimentación y Cultura (pp. 27-81). Barcelona: España: Universidad de Barcelona.

Milton, K. (1993). Dieta y evolución de los primates. Investigación y Ciencia , 205, 56-63.

Mithen, S. (1996). Arqueología de la mente . Barcelona, España: Grijalbo-Monadori.

Ribes, E. (1992). Sobre el tiempo y el espacio psicológicos. Acta Comportamentalia , 1, 71-84.

Ribes, E. (1998). Teoría de la conducta: logros, avances y tareas pendientes. Acta Comportamentalia , 6 (Monográfico), 127-147.

Ribes, E. (2004). Acerca de las funciones psicológicas: un postscriptum. Acta Comportamentalia , 12, 117-127.

Ribes, E. (2007). Estados y límites del campo, medios de contacto y análisis molar del comportamiento: reflexiones teóricas. Acta Comportamentalia , 15, 229-259.

Ribes, E. (2009). Reflexiones sobre la aplicación del conocimiento psicológico: ¿Qué aplicar 
o cómo aplicar? Revista Mexicana de Análisis de la Conducta , 35, 3-17.

Ribes, E., \& López. (1985). Teoría de la conducta. México: Trillas.

Ross, E. (1995). Una revisión de las tendencias dietéticas desde los cazadores- recolectores hasta las sociedades capitalistas modernas. En J. Contreras (Ed.), Alimentación $y$ cultura (pp. 259-306). Barcelona: Universitat de Barcelona.

Rozin, P. (1995). Perspectivas psicobiológicas sobre las preferencias y aversiones alimentarias. En J. Contreras (Ed.), Alimentación y cultura (pp. 85-109). Barcelona: Universitat de Barcelona.

Tutin, C., Fernández, M., Rogers, M. E., Williamson, E. A., \& McGrew, W. C. (1992). Foraging profiles of sympatric lowland gorillas and chimpanzees in the Lopé Reserve, Gabon. En A. Whiten \& E. M. Widdowson (Eds.), Foraging strategies and natural diet of monkeys, apes and humans (pp. 19-25). Nueva York: Clarendon Press Oxford.

Ulijaszek, S. J. (1992). Human dietary change. En A. Whiten \& E. M. Widdowson (Eds.), Foraging strategies and natural diet of monkeys, apes and humans (pp. 111-118). Nueva York: Clarendon Press Oxford.

Whiten, A., Byrne, R. W., Barton, R. A., Waterman, P. G., \& Henzi, S. P. (1992). Dietary and foraging strategies of baboons. En A. Whiten \& E. M. Widdowson (Eds.), Foraging strategies and natural diet of monkeys, apes and humans (pp. 27-35). Nueva York:Clarendon Press Oxford.

\section{Notas}

* Artículo de investigación 